\title{
Indications for single-dose activated charcoal administration in acute overdose
}

\author{
Geoffrey K. Isbister ${ }^{\mathrm{a}}$ and Venkata V. Pavan Kumar ${ }^{\mathrm{b}}$
}

aDepartment of Clinical Toxicology and Pharmacology, Calvary Mater Newcastle and Discipline of Clinical Pharmacology, University of Newcastle, New South Wales, Australia and ${ }^{\mathrm{b}}$ School of Pharmacy, University of Otago, Otago, New Zealand

Correspondence to Geoffrey K. Isbister, Department of Clinical Toxicology and Pharmacology, Calvary Mater Newcastle, Edith St, Waratah, NSW 2298, Australia Tel: +6124921 1211; fax: +6124921 1870; e-mail: geoff.isbister@gmail.com

Current Opinion in Critical Care 2011, 17:351-357

\section{Purpose of review}

Gastrointestinal decontamination in overdose patients remains a controversial problem in emergency medicine. There has been a significant decrease in the use of single-dose activated charcoal (SDAC) in recent years based on little new evidence and possibly because the overall mortality in overdose patients is low.

\section{Recent findings}

Human volunteer studies suggest SDAC is effective and this effect occurs for up to $4 \mathrm{~h}$ after ingestion, but the magnitude of the reduction in area under the curve (AUC) decreases over time. Two randomized controlled trials including one recent large study did not find SDAC to be beneficial. Pharmacokinetic and pharmacodynamic studies of specific drugs in overdose suggest that for most drugs SDAC decreases drug exposure, but this does not translate to clinical benefit in all cases. The administration of SDAC is a low-risk intervention.

\section{Summary}

Although SDAC is unlikely to be beneficial in many overdose patients, for some subgroups with severe poisoning, the benefits will outweigh the low risk of administration. The use of SDAC should be based on the potential toxicity of the drug ingested and the potential benefit of SDAC balanced against the willingness of the patient to take SDAC and the low risk of administration.

\section{Keywords}

activated charcoal, decontamination, drug overdose, pharmacokinetic, poisoning

\section{Introduction}

Gastrointestinal decontamination in overdose patients remains a controversial problem in emergency medicine. Activated charcoal nonselectively binds to most mediumsized carbon-based poisons (such as pharmaceuticals). There has been a significant decrease in the use of activated charcoal in recent years based on little new evidence and possibly because the overall mortality in overdose patients is low. A position statement published in 1997 questioned the use of single-dose activated charcoal (SDAC) more than an hour after ingestion which contributed to a substantial decrease in its use [1]. This position statement was revised in 2005 but little research had been undertaken during this time and its recommendations therefore remained unchanged $\left[2^{\bullet}, 3\right]$. Recently a large RCT of over 4500 patients in Sri Lanka showed no advantage of SDAC or multiple-dose activated charcoal in acute poisoning $\left[4^{\bullet}\right]$. However, this study included a large proportion of pesticide and oleander seed poisonings with very different absorption kinetics to most prescription medications. In addition, the accompanying editorial cautioned against generalizing this study to settings outside the rural tropics and asked for robust pharmacokinetics studies to better understand the role of SDAC in poisoning [5].

The lack of evidence to support SDAC and the low mortality in overdose patients, particularly with most prescription medications, suggests that for the majority, SDAC is probably not warranted. However, the question remains as to whether there is a subgroup of patients with severe poisoning in which SDAC will be beneficial. Currently the use of SDAC in severe poisoning is ad hoc and in most cases has little scientific basis or support from clinical trials. Even for less severe poisoning there may be benefits from SDAC in terms of reduced need for life support, monitoring and other antidotes. Previous controlled trials have included heterogeneous groups of overdose patients $\left[4^{\bullet}, 6^{\bullet}\right]$, often excluding the most severe poisoning, so they are unlikely to detect benefit in small subgroups of patients taking individual drugs or suffering severe poisoning. There has been some recent work that has attempted to quantify the effects of SDAC for individual drugs $\left[7,8^{\bullet}, 9,10^{\bullet}, 11,12\right]$ which demonstrate variable efficacy of SDAC on the pharmacokinetics of 
drugs in overdose and have shown effectiveness against clinical outcomes for some drugs but not others. Some of these and previous studies provide enough information to allow a decision to be made about the use of SDAC for specific drugs for individual patients $\left[8^{\bullet}, 10^{\bullet}, 13^{\bullet}, 14\right]$.

The aim of this article is to review the recent literature in light of changes in the use of activated charcoal over the last two decades and provide an approach to applying this information to individual overdose patients. The review will focus on the initial use of activated charcoal, SDAC.

\section{Activated charcoal in human volunteers}

Studies in human volunteers provide the basis for activated charcoal being used in overdose patients, including the effect of time of administration of SDAC, the amount of activated charcoal and the role of the volume of distribution of the ingested drugs $\left[2^{\bullet}, 15^{\bullet}, 16^{\bullet \bullet}\right]$. Figure 1 provides a scatter plot of the human volunteer studies included in the position statement in 2005 [2*]. The figure shows that SDAC is effective in human volunteer studies and this effect occurs for up to $4 \mathrm{~h}$ after ingestion but the magnitude of the reduction in area under the curve (AUC) decreases over time. This is similar to a meta-analysis undertaken by Jurgens et al. [15].

Human volunteer studies have measured the effect of SDAC on the AUC of the drug and although this is most commonly interpreted as a reduction in the absorption of drug, it may also be due to an increase in clearance or a combination of both increase in clearance and decrease in absorption. This is seen in Eq. (1).

$\mathrm{AUC}=\frac{\text { Dose } \times F}{\mathrm{CL}}$

\section{Key points}

- Single dose activated charcoal is unlikely to be beneficial in the majority of overdose patients.

- There is probably benefit of activated charcoal in a subgroup of patients with severe poisoning.

- The decision to give activated charcoal should be based on ingested drug toxicity and potential benefit of charcoal, balanced against the willingness of the patient to take charcoal and its low risk of administration.

AUC is directly proportional to the bioavailability $(F)$ and inversely proportional to clearance (CL). Hence either an increase in clearance or a reduction in bioavailability can decrease the AUC. Overall the change in the AUC that was seen in all the above studies can only be determined to the level of the ratio of bioavailability and clearance as shown in Eq. (2).

$\frac{\mathrm{AUC}}{\mathrm{Dose}}=\frac{F}{\mathrm{CL}}$

Although studies of SDAC conducted in the healthy volunteers support the hypothesis that SDAC reduces AUC and hence drug exposure, they do not determine if this is due to a decrease in fraction absorbed or due to an increase in clearance, or if extraneous variables or covariates affect this. One recent study in human volunteers given supratherapeutic doses $(50 \mathrm{mg} / \mathrm{kg})$ of acetaminophen found that increasing doses of SDAC decreased the half-life of acetaminophen from $2.5 \mathrm{~h}$ for a $5 \mathrm{~g}$ dose of SDAC to $1.9 \mathrm{~h}$ and $1.6 \mathrm{~h}$ for 25 and $50 \mathrm{~g}$ of SDAC, respectively, which was statistically significant [17]. This supports that SDAC can also increase clearance and perhaps this effect is more important with larger doses of SDAC which can absorb larger amounts of drug.

Figure 1 Summary of the human volunteer studies from the position statement on single dose activated charcoal

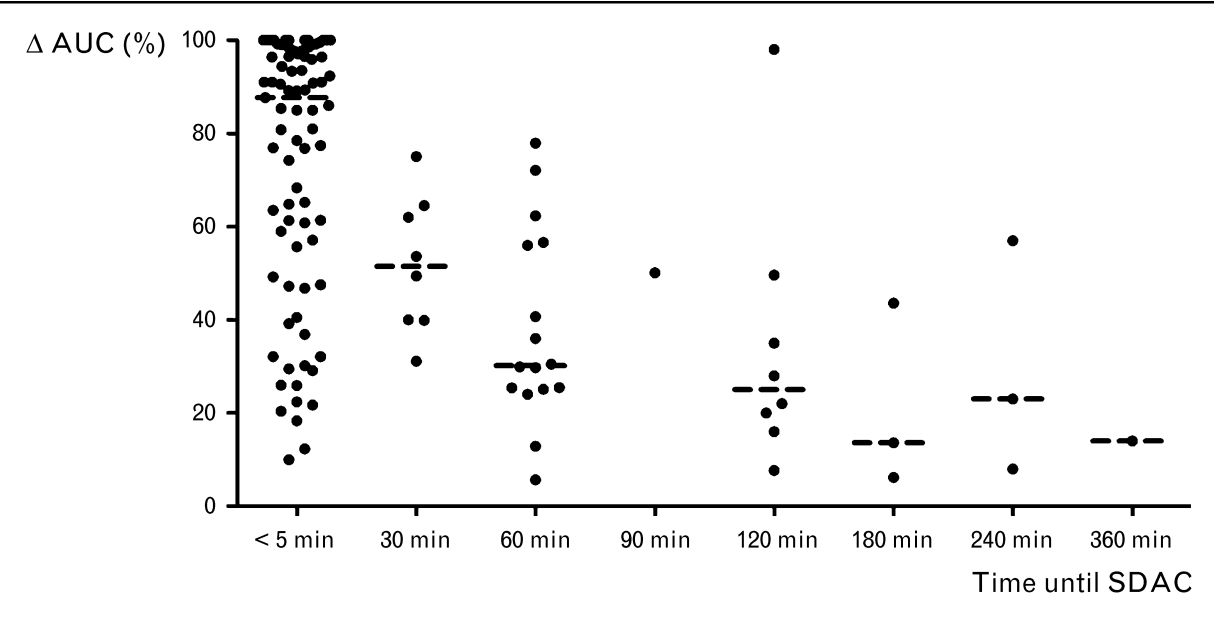


The meta-analysis by Jurgens $e t$ al. $\left[15^{\circ}\right]$ also showed that the effect of SDAC increases with the dose of charcoal given. However, the recent human volunteer study of paracetamol ingestions only provided weak evidence to support this and that a recommendation of a 10:1 ratio of SDAC to ingested drug mass appears to be reasonable. The reduction in AUC due to SDAC is also associated with the volume of distribution of the ingested drug and is greater with drugs with large volumes of distribution $\left[15^{\circ}\right]$.

\section{Evidence from pharmacokinetic and pharmacodynamics studies in overdose patients}

Human volunteer studies continue to be done with SDAC and although more recent studies have used supratherapeutic amounts of drug [17-19], they are still far below the amounts ingested by most overdose patients and only provide information on the pharmacokinetics in therapeutic or supratherapeutic amounts. What is required are studies of the effect of SDAC on the pharmacokinetics of drugs in overdose and more importantly if the effect of SDAC on the pharmacokinetics translates to an effect on the pharmacodynamics of drugs in overdose, that is of clinical benefit.
A series of studies on citalopram overdose explored the effect of SDAC on the pharmacokinetics and pharmacodynamics of citalopram, to attempt to develop clinical guidelines. The initial study was a population pharmacokinetic study of citalopram overdoses that showed that SDAC almost doubled the clearance and reduced the fraction absorbed by $22 \%$ and in the study the majority of patients received SDAC 2-4 h after ingestion [20]. The second study of the pharmacodynamics of citalopram in overdose showed that this effect of SDAC on the pharmacokinetics translated into a reduced probability of the patient developing an abnormal QT [7]. Using the pharmacokinetic-pharmacodynamic model developed, simulations were then used to explore a range of patient presentations based on dose ingested, SDAC and the QT interval to propose clinical guidelines for citalopram overdoses (Fig. 2) [21 $\left.{ }^{\circ}\right]$. Finally a second dataset of patients was used to test the assertion that SDAC reduced the probability of $\mathrm{QT}$ prolongation in citalopram overdoses $\left[8^{\bullet}\right]$.

Further studies have now been undertaken for other overdoses, including quetiapine and venlafaxine. These have demonstrated two important findings: 1) that different drugs are affected by SDAC in different ways; and 2) that a reduction in drug exposure may not necessarily translate into a clinical benefit. This was best seen with

Figure 2 Clinical recommendations for the management of citalopram overdose including the use of single-dose activated charcoal based on the risk of QT prolongation

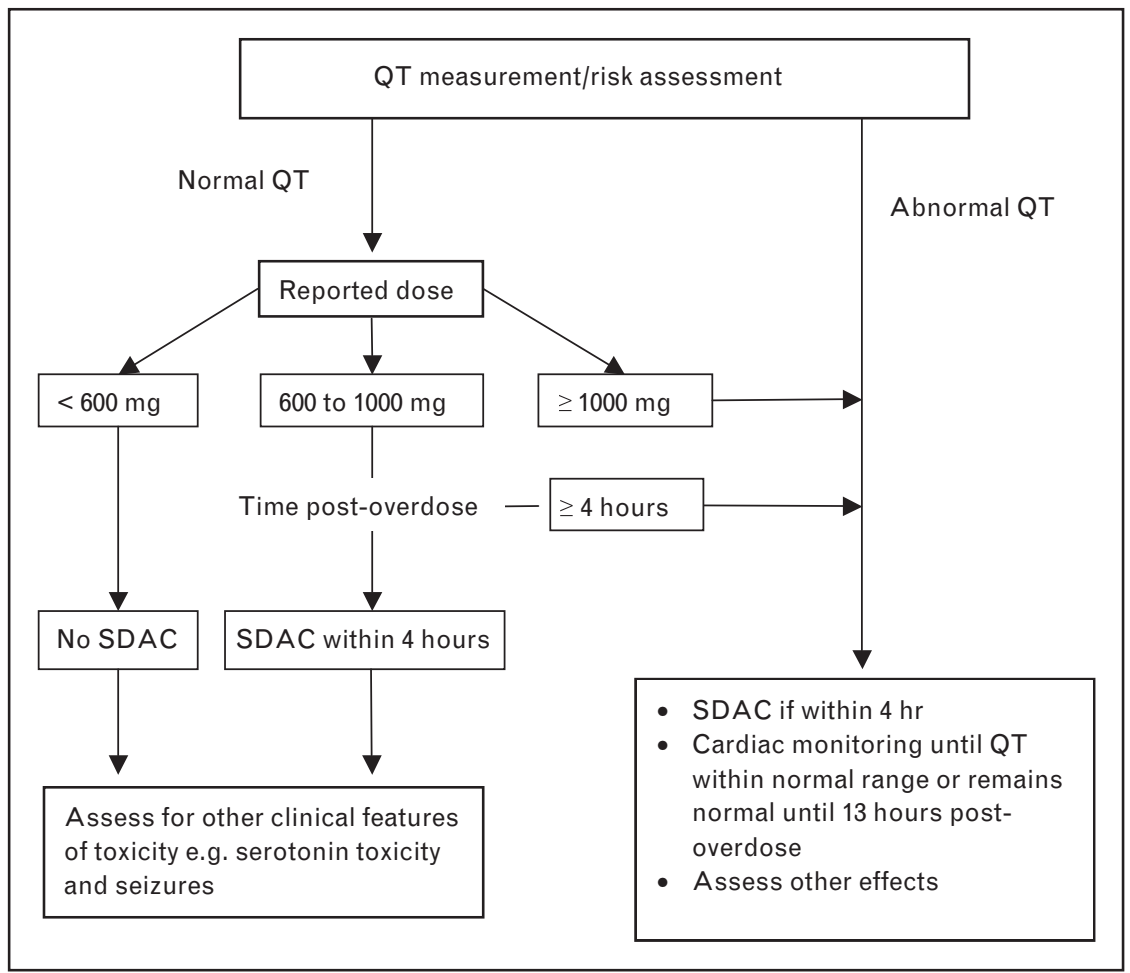


the studies of quetiapine overdose in which in the first study SDAC reduced the fraction absorbed by over a third, but had no effect on clearance [9]. This is probably because SDAC is only likely to increase the clearance of drugs with long half-lives such as citalopram (median $40 \mathrm{~h}$ in overdose [20]) and not ones with short half-lives like quetiapine (median $6.6 \mathrm{~h}$ in overdose [9]). The second study showed that SDAC was unlikely to have a major impact on clinical outcomes in quetiapine overdose, including requirement for intubation and duration of mechanical ventilation, so the use of SDAC is unlikely to be beneficial $\left[10^{\bullet}\right]$.

Quantitative pharmacokinetic and pharmacodynamics studies therefore provide an approach to investigating the effect of SDAC for individual drugs in overdose. These studies are generally regarded as exploratory or hypothesis-generating studies and focused controlled trials can then be undertaken to confirm the effects $\left[8^{\bullet}, 22\right]$. Further investigation with other drugs that demonstrate clinically significant toxicity is warranted.

\section{Evidence from controlled trials of clinical outcomes in overdose patients}

Although a number of controlled trials of decontamination in acute overdose have been undertaken there are only two well designed randomized controlled trials that have compared SDAC to supportive care alone and neither demonstrated clinical benefit of charcoal $\left[4^{\bullet}, 6^{\bullet}, 23,24\right]$. The first trial by Cooper et al. included a large number of cases which were unlikely to develop significant toxicity and would have therefore had good outcomes irrespective of SDAC. Seven patients with severe toxicity were excluded $\left[6^{\bullet}\right]$. The second and largest trial of activated charcoal by Eddleston et al. did not exclude patients based on severity and also did not show an effect of SDAC. This study mainly included pesticides and oleander so it is difficult to apply these findings to prescription drugs $\left[4^{\circ}\right]$. These studies and other studies that have included activated charcoal have been reviewed in detail elsewhere $\left[16^{\bullet \bullet}, 25^{\bullet}\right]$.

A number of studies have investigated the effects of activated charcoal on acetaminophen poisoning. The first study compared three different decontamination methods in patients ingesting $5 \mathrm{~g}$ or more acetaminophen and showed that SDAC decreased the absorption of acetaminophen [26]. The second was a retrospective analysis of 981 acetaminophen overdoses which demonstrated that administration of SDAC within $2 \mathrm{~h}$ reduced the number of patients with a toxic acetaminophen concentration $\left[13^{\circ}\right]$. Two other studies also suggest a beneficial effect of SDAC in acetaminophen poisoning $[27,28]$ and collectively these studies would suggest that SDAC has a role in acetaminophen poisoning. However, the exact role including dose of SDAC and timing needs further delineation.

Three studies were unable to show any benefit on plasma drug concentrations or clinical outcomes in tricyclic antidepressant overdose, including a direct comparison of SDAC versus supportive care and another comparing SDAC in patients having gastric lavage against gastric lavage alone [29-31]. However, these studies were small and did not undertake a formal pharmacokinetic analysis of the data.

Unfortunately it is difficult to draw any conclusions from all of these studies testing SDAC because in large studies of heterogeneous patient groups any effect on small subgroups may be missed. However, the apparent benefit of SDAC in acetaminophen poisoning and conversely the lack of significant benefit in studies of tricyclic antidepressant poisoning do suggest that for some drugs there is a potential benefit and further studies of drugs with significant effects should be undertaken.

\section{Risks of administration of activated charcoal administration}

The administration of activated charcoal is a low-risk procedure. However, the administration of activated charcoal is associated with considerable unpleasantness because it is distasteful to drink. It is frequently blamed for causing an increased risk of vomiting but this is not supported by the controlled trial of Cooper $e t a l$. in which there was no difference in the frequency of vomiting between SDAC and no decontamination $\left[6^{\circ}\right]$ and vomiting was not reported in the largest trial of activated charcoal $\left[4^{\bullet}, 32\right]$.

The most serious potential complication in overdose patients is aspiration pneumonitis and there are case reports of severe cases of aspiration pneumonia reported in association with activated charcoal administration or confirmation of activated charcoal in the lungs [33]. There are two separate issues in regards to SDAC whether SDAC increases the risk of aspiration and whether aspiration of activated charcoal in addition to gastric contents worsens the aspiration pneumonitis. A large study of overdose patients which investigated the risk factors for aspiration pneumonitis did not identify SDAC as a risk factor $\left[34^{\circ}\right]$. In addition the large trial of activated charcoal in Sri Lanka did not identify aspirated charcoal in any deaths which would have included cases of aspiration pneumonitis $\left[4^{\bullet}\right]$.

It has also been suggested that aspiration of activated charcoal is worse than aspiration of gastric contents alone. This is based on animal studies in which activated charcoal is instilled into the lungs of rats which results in 
pulmonary injury compared to sterile water [35]. However, these studies are unlikely to represent aspiration of activated charcoal in human poisoning when activated charcoal will be aspirated with gastric contents, so a more appropriate animal study would be instilling gastric contents with and without activated charcoal. There is therefore little evidence to support the use of SDAC increasing the risk of aspiration or worsening the severity of aspiration pneumonitis.

Rarer complications of activated charcoal include bowel obstruction, and this has been reported in two cases in the Sri Lankan study $\left[4^{\bullet}\right]$. This may be a concern for drugs with anticholinergic effects but are usually associated with multiple-dose activated charcoal [36]. Although SDAC is not a completely risk-free intervention it can be regarded as a low-risk procedure that should be considered whenever there is potential benefit in severe poisoning.

\section{Approaching the individual patient}

The decision to give a patient activated charcoal is an excellent example of the difference between what a clinician must decide to do with an individual patient and what the evidence shows. If a decision is based on controlled trial evidence then there is little to support the benefit of SDAC. However, the quantitative studies and evidence in small studies of select drugs suggest there is potential benefit in some situations and there is a role for SDAC in acute overdose.
Previous reviews have discussed various approaches to deciding whether an individual patient will benefit from the administration of activated charcoal [37,38]. Bailey [38] discussed a triangle of three things - potential toxicity of the poison, the benefit of SDAC, balanced against the risks of charcoal. These are the essential issues that the clinician needs to resolve before giving SDAC, but they are not really in a triangle but a series of questions the clinician must ask. The potential toxicity of the overdose must be considered first and if there is no potential toxicity then any form of decontamination is not required (Fig. 3). If the clinician does not believe the patient is at risk of any complications from the poisoning then there is no reason to give SDAC. Part of this consideration also needs to be the potential toxicity based on the dose, the time since the overdose: Have we reached the peak effect already? Will this dose cause toxicity? If the clinician determines there is potential toxicity, the potential benefit of SDAC for the ingested drug can then be balanced against the risks of administration.

\section{Practicalities and ethics of activated charcoal administration}

The administration of activated charcoal is different to other medications because it is unpleasant to drink and in many cases the patient may not be cooperative or may simply refuse to drink it [32,39]. There is therefore an additional consideration of the willingness of the patient

Figure 3 A proposed decision-making process for the administration of single-dose activated charcoal in overdose patients

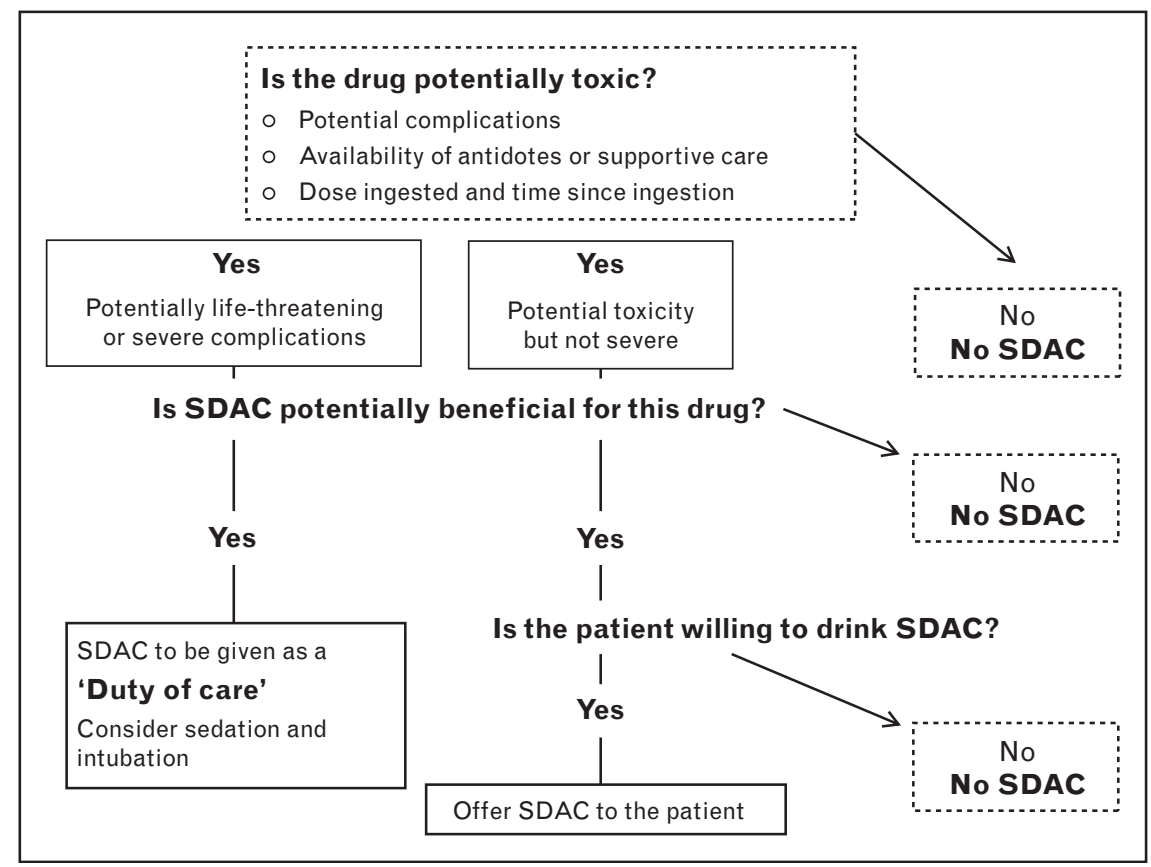


to drink activated charcoal, which will need to be balanced against the benefit (Fig. 3). It could be argued that this is more important than the risk of administration because SDAC is a low-risk intervention. If the patient is willing to drink charcoal then the decision to administer can be simply based on the potential benefit. If the patient is unconscious then as long as the airway is protected the decision to administer SDAC is the same. However, it is difficult, if not impossible, to administer SDAC in a noncooperative awake patient. The use of physical restraint and a nasogastric or orogastric tube is no longer acceptable, raises numerous ethical concerns and has legal implications, depending on local law. Worse are attempts to give activated charcoal in patients who have an altered mental state, either delirium or a decreased level of consciousness. Although these patients may not need airway protection for the poisoning, administering SDAC is difficult and contra-indicated unless they have airway protection.

Therefore, the awake noncooperative patients and patients with altered mental state pose a unique problem in deciding to give SDAC. There needs to be significant potential benefit of activated charcoal if it is going to be administered because these patients are going to require sedation and intubation for airway protection, so that SDAC can be safely administered. This adds another level of complexity to the decision-making process to administer SDAC and the clinician needs to answer the question: 'Do I believe that the overdose is likely to cause life-threatening toxicity, that is not easily treated by supportive care and/or a specific antidote, that I am willing to intubate this patient against their will to administer activated charcoal?' This may be clear-cut, for example, in a patient presenting early after a large colchicine overdose which is life-threatening and decontamination may be the only beneficial treatment. However, for an acetaminophen overdose in which there is some evidence to support the use of SDAC [13 $\left.{ }^{\bullet}\right]$, it is unlikely to be sufficient to intubate a patient because administration of $\mathrm{N}$-acetylcysteine within $8 \mathrm{~h}$ is a highly effective treatment.

It is possible to consider two groups of patients in which SDAC is beneficial (Fig. 3). One group are cooperative patients in which administration may improve intermediate outcomes such as reduce length of hospital stay or prevent the need for the administration of an antidote. Examples of this group are acetaminophen poisoning or sedative drug poisoning which can be managed with supportive care. The other group of patients is those in which there is life-threatening poisoning that is not easily treated, such as arrhythmias, multiorgan failure, when even a small benefit of SDAC may improve outcomes. In this group it is a duty of care to administer SDAC, although the practicalities of this will differ locally.

\section{Conclusion}

Hopefully future studies will focus on investigating the benefits of SDAC in subgroups of patients with severe poisoning from specific drugs and methods of quantitative analysis, both pharmacokinetics and pharmacodynamics, may be employed [40]. SDAC must remain an important consideration in the treatment of severe poisoning but clearly is not required in the majority of overdose patients.

\section{Acknowledgement}

G.K.I. is supported by an NHMRC Clinical Career Development Award ID605817.

\section{References and recommended reading}

Papers of particular interest, published within the annual period of review, have been highlighted as:

- of special interest

-• of outstanding interest

Additional references related to this topic can also be found in the Current World Literature section in this issue (pp. 403-404).

1 Chyka PA, Seger D. Position statement: single-dose activated charcoal. American Academy of Clinical Toxicology; European Association of Poisons Centres and Clinical Toxicologists. J Toxicol Clin Toxicol 1997; 35:721-741.

2 Chyka PA, Seger D, Krenzelok EP, Vale JA. Position paper: single-dose - activated charcoal. ClinToxicol (Phila) 2005; 43:61-87.

Consensus statement of the North American and European Associations of Poison Centres and Clinical Toxicology.

3 Buckley NA, Eddleston M. The revised position papers on gastric decontamination. ClinToxicol (Phila) 2005; 43:129-130.

4 Eddleston M, Juszczak E, Buckley NA, et al. Multiple-dose activated charcoal

- in acute self-poisoning: a randomised controlled trial. Lancet 2008; 371 579-587.

Largest randomized controlled trial of activated charcoal that shows no difference between single dose activated charcoal, multiple dose activated charcoal and supportive care in over $\mathbf{4 5 0 0}$ patients mainly ingesting pesticides and oleander.

5 Eyer $\mathrm{P}$, Eyer $\mathrm{F}$. Is this the epitaph for multiple-dose activated charcoal? Lancet 2008; 371:538-539.

6 Cooper GM, Le Couteur DG, Richardson D, Buckley NA. A randomized

- clinical trial of activated charcoal for the routine management of oral drug overdose. QJM 2005; 98:655-660.

Only well-designed randomized controlled trial of single dose activated charcoal versus supportive care with mainly prescription medication overdoses that showed no difference. It also showed that activated charcoal did not increase the frequency of vomiting.

7 Friberg LE, Isbister GK, Duffull SB. Pharmacokinetic-pharmacodynamic modelling of OT interval prolongation following citalopram overdoses. $\mathrm{Br} \mathrm{J}$ Clin Pharmacol 2006; 61:177-190.

8 Isbister GK, Friberg LE, Stokes B, et al. Activated charcoal decreases the risk

- of OT prolongation after citalopram overdose. Ann Emerg Med 2007; 50:593-600.

Large study of citalopram overdoses that demonstrated that single dose activated charcoal reduced the probability of developing an abnormal QT. The study used a retrospective dataset and incorporated prior information from a pharmacokineticpharmacodynamic study using a fully Bayesian analysis.

9 Isbister GK, Friberg LE, Hackett LP, Duffull SB. Pharmacokinetics of quetiapine in overdose and the effect of activated charcoal. Clin Pharmacol Ther 2007; 81:821-827.

10 Isbister GK, Duffull SB. Quetiapine overdose: predicting intubation, duration - of ventilation, cardiac monitoring and the effect of activated charcoal. Int Clin Psychopharmacol 2009; 24:174-180.

Study of quetiapine overdoses investigating the effect of activated charcoal on requirement for intubation and duration of mechanical ventilation. Although there was a marginal benefit of activated charcoal reducing the probability of intubation if given within $2 \mathrm{~h}$, it had no effect on duration of ventilation.

11 Kumar VV, Oscarsson S, Friberg LE, et al. The effect of decontamination procedures on the pharmacokinetics of venlafaxine in overdose. Clin Pharmacol Ther 2009; 86:403-410. 
12 Kumar VPK, Geoffrey KI, Duffull SB. The effect of decontamination procedures on pharmacodynamics of venlafaxine in overdose. Br J Clin Pharmacol 2011. doi: 10.1111/j.1365-2125.2011.03934.x.

13 Buckley NA, Whyte IM, O'Connell DL, Dawson AH. Activated charcoal

- reduces the need for $\mathrm{N}$-acetylcysteine treatment after acetaminophen (paracetamol) overdose. J Toxicol Clin Toxicol 1999; 37:753-757.

Large retrospective study of acetaminophen overdose that found that giving activated charcoal within 2 hours reduced the number of patients with toxic acetaminophen concentrations.

14 Page CB, Duffull SB, Whyte IM, Isbister GK. Promethazine overdose: clinical effects, predicting delirium and the effect of charcoal. QJM 2009; 102:123131.

15 Jurgens G, Hoegberg LC, Graudal NA. The effect of activated charcoal on

- drug exposure in healthy volunteers: a meta-analysis. Clin Pharmacol Ther 2009; 85:501-505.

Meta-analysis of trials of activated charcoal in human volunteers showing that single dose activated charcoal reduced the AUC and this effect occurred for up to $4 \mathrm{~h}$ after ingestion although the magnitude of the effect decreased over time.

16 Olson KR. Activated charcoal for acute poisoning: one toxicologist's journey.

-• J Med Toxicol 2010; 6:190-198.

Recent review on activated charcoal for acute poisoning that provides a more indepth review of some parts of the subject.

17 Gude AB, Hoegberg LC, Angelo HR, Christensen HR. Dose-dependent adsorptive capacity of activated charcoal for gastrointestinal decontamination of a simulated paracetamol overdose in human volunteers. Basic Clin Pharmacol Toxicol 2010; 106:406-410.

18 Keranen T, Sorri A, Moilanen E, Ylitalo P. Effects of charcoal on the absorption and elimination of the antiepileptic drugs lamotrigine and oxcarbazepine. Arzneimittelforschung 2010; 60:421-426.

19 Wananukul W, Klaikleun S, Sriapha C, Tongpoo A. Effect of activated charcoal in reducing paracetamol absorption at a supra-therapeutic dose. J Med Assoc Thai 2010; 93:1145-1149.

20 Friberg LE, Isbister GK, Hackett LP, Duffull SB. The population pharmacokinetics of citalopram after deliberate self-poisoning: a Bayesian approach. J Pharmacokinet Pharmacodyn 2005; 32:571-605.

21 Isbister GK, Friberg LE, Duffull SB. Application of pharmacokinetic-pharma-

- codynamic modelling in management of QT abnormalities after citalopram overdose. Intensive Care Med 2006; 32:1060-1065.

Simulation study that used a previously developed pharmacokinetic-pharmacodynamic study of citalopram overdoses and the effect of single dose activated charcoal to develop an approach to managing citalopram overdose based on quantitative analysis.

22 Sheiner LB. Learning versus confirming in clinical drug development. Clin Pharmacol Ther 1997; 61:275-291.

23 Merigian KS, Woodard M, Hedges JR, et al. Prospective evaluation of gastric emptying in the self-poisoned patient. Am J Emerg Med 1990; 8:479-483.

24 Merigian KS, Blaho KE. Single-dose oral activated charcoal in the treatment of the self-poisoned patient: a prospective, randomized, controlled trial. Am J Ther 2002; 9:301-308.
25 Bond GR. The role of activated charcoal and gastric emptying in gastro- intestinal decontamination: a state-of-the-art review. Ann Emerg Med 2002; 39:273-286.

State of the art review of activated charcoal and gastric emptying in overdose patients.

26 Underhill TJ, Greene MK, Dove AF. A comparison of the efficacy of gastric lavage, ipecacuanha and activated charcoal in the emergency management of paracetamol overdose. Archives of Emergency Medicine 1990; 7:148-154.

27 Spiller HA, Sawyer TS. Impact of activated charcoal after acute acetaminophen overdoses treated with N-acetylcysteine. J Emerg Med 2007; 33:141144.

28 Spiller HA, Winter ML, Klein-Schwartz W, Bangh SA. Efficacy of activated charcoal administered more than four hours after acetaminophen overdose. J Emerg Med 2006; 30:1-5.

29 Crome $\mathrm{P}$, Adams R, Ali C, et al. Activated charcoal in tricyclic antidepressant poisoning: pilot controlled clinical trial. Hum Toxicol 1983; 2:205-209.

30 Bosse GM, Barefoot JA, Pfeifer MP, Rodgers GC. Comparison of three methods of gut decontamination in tricyclic antidepressant overdose. J Emerg Med 1995; 13:203-209.

31 Hulten BA, Adams R, Askenasi R, et al. Activated charcoal in tricyclic antidepressant poisoning. Hum Toxicol 1988; 7:307-310.

32 Mohamed F, Sooriyarachchi MR, Senarathna L, et al. Compliance for single and multiple dose regimens of superactivated charcoal: a prospective study of patients in a clinical trial. Clin Toxicol (Phila) 2007; 45:132-135.

33 Seger D. Single-dose activated charcoal: backup and reassess. J Toxicol Clin Toxicol 2004; 42:101-110.

34 Isbister GK, Downes F, Sibbritt D, et al. Aspiration pneumonitis in an

- overdose population: frequency, predictors, and outcomes. Crit Care Med $2004 ; 32: 88-93$.

Study of a large number of overdose patients that investigates the predictors of aspiration in overdose patients which included a decreased level of consciousness, but did not include activated charcoal.

35 Arnold $\mathrm{TC}$, Willis $\mathrm{BH}$, Xiao $\mathrm{F}$, et al. Aspiration of activated charcoal elicits an increase in lung microvascular permeability. Journal of Toxicology Clinical Toxicology 1999; 37:9-16.

36 Chan JC, Saranasuriya C, Waxman BP. Bezoar causing small bowel obstruction after repeated activated charcoal administration. Med J Aust 2005; 183:537.

37 Greene S, Harris C, Singer J. Gastrointestinal decontamination of the poisoned patient. Pediatr Emerg Care 2008; 24:176-186; quiz 87-9.

38 Bailey B. Gastrointestinal decontamination triangle. Clin Toxicol (Phila) 2005; 43:59-60.

39 Alaspaa AO, Kuisma MJ, Hoppu K, Neuvonen PJ. Out-of-hospital administration of activated charcoal by emergency medical services. Ann Emerg Med 2005; 45:207-212.

40 Isbister GK. How do we use drug concentration data to improve the treatment of overdose patients? Ther Drug Monit 2010; 32:300-304. 